\title{
The role of TFPI2 hypermethylation in the detection of gastric and colorectal cancer
}

\author{
Haochang $\mathrm{Hu}^{1, *}$, Xiaoying Chen ${ }^{1, *}$, Cheng Wang ${ }^{2, *}$, Yuting Jiang ${ }^{1}$, Jingjing Li ${ }^{3}$, Xiuru \\ Ying ${ }^{1}$, Yong Yang ${ }^{1}$, Bin $\mathrm{Li}^{1}$, Cong Zhou ${ }^{1}$, Jie Zhong ${ }^{1}$, Dongping $\mathrm{Wu}^{2}$, Jieer Ying ${ }^{3}$ and \\ Shiwei Duan ${ }^{1}$ \\ ${ }^{1}$ Medical Genetics Center, School of Medicine, Ningbo University, Ningbo, Zhejiang 315211, China \\ ${ }^{2}$ Department of Medical Oncology, Shaoxing People's Hospital, Shaoxing Hospital of Zhejiang University, Zhejiang 312000, \\ China \\ ${ }^{3}$ Department of Medical Oncology, Zhejiang Cancer Hospital, Hangzhou, Zhejiang 310022, China \\ *Co-first authors
}

Correspondence to: Shiwei Duan, email: duanshiwei@nbu.edu.cn Jieer Ying, email: jieerying@aliyun.com

Keywords: tissue factor pathway inhibitor 2, DNA methylation, gastric cancer, colorectal cancer, quantitative methylation-specific polymerase chain reaction

Received: November 30, $2016 \quad$ Accepted: August 28, 2017

Published: September 20, 2017

Copyright: Hu et al. This is an open-access article distributed under the terms of the Creative Commons Attribution License 3.0 (CC BY 3.0 ), which permits unrestricted use, distribution, and reproduction in any medium, provided the original author and source are credited.

\section{ABSTRACT}

Gastrointestinal cancer is a prevalent disease with high morbidity and mortality. Tissue factor pathway inhibitor 2 (TFPI2) gene could protect the extracellular matrix of cancer cells from degradation and tumor invasion. The goal of our study was to estimate the diagnostic value of TFPI2 hypermethylation in gastric cancer (GC) and colorectal cancer (CRC). TFPI2 methylation was measured by quantitative methylation-specific polymerase chain reaction (qMSP) method in 114 GC and 80 CRC tissues and their paired non-tumor tissues. Our results showed that TFPI2 methylation was significantly higher in tumor tissues (GC: $29.940 \%$ vs. $12.785 \%$, $P<0.001$; CRC: $26.930 \%$ vs. $5.420 \%, P<0.001$ ). The methylation level of TFPI2 in colorectal tumor tissues was significantly higher than that in colorectal normal tissues (26.930\% versus $0.002 \%, P<0.00001)$. In GC, TFPI2 hypermethylation yielded an area under the curve (AUC) of 0.762 (95\% CI: $0.696-0.828)$ with a sensitivity of $68 \%$ and a specificity of $83 \%$. In CRC, TFPI2 hypermethylation yielded an AUC of 0.759 (95\% CI: $0.685-0.834$ ) with a sensitivity of $61 \%$ and a specificity of $84 \%$. Similarly, TCGA data also supported TFPI2 hypermethylation was a promising diagnostic marker for GC and CRC. Moreover, the dual-luciferase reporter assay showed TFPI2 fragment could upregulate gene expression (fold change $=5, P=0.005$ ). Data mining further indicated that TFPI2 expression in CRC cell lines was significantly increased after 5'-AZA-deoxycytidine treatment (fold change > 1.37). In conclusion, TFPI2 hypermethylation might be a promising diagnostic biomarker for GC and CRC.

\section{INTRODUCTION}

The gastrointestinal tract is one of the most predilection sites of tumorigenesis [1]. In China, about 679,100 cases of gastric cancer (GC) and 376,300 cases of colorectal cancer (CRC) were diagnosed in 2015 [2]. Despite the rapid improvement in clinical therapy, GC and $\mathrm{CRC}$ are still global public health problems due to their poor prognosis [3, 4]. Lacking a gold standard of noninvasive method is considered as the major obstacle to the screening, diagnosis and individualized treatment of GC and CRC. For this purpose, it is urgent to identify suitable biomarkers for the early detection of $\mathrm{GC}$ and CRC. 
The development of GC and CRC is characterized by the dysregulation of genetic, epigenetic, and environmental factors $[5,6]$. As a bridge between the genetic and environmental factors, epigenetic modification plays an important role in cancer initiation and progression. DNA methylation is acknowledged as the principle mechanism of dysfunction of tumor suppressor genes [7] and the activation of oncogenes [8]. DNA methylation has been widely studied in GC and CRC [9-12].

Tissue factor pathway inhibitor 2 (TFPI2) gene is located on chromosome $7 q 22$, encoding a Kunitz-type serine proteinase inhibitor [13]. TFPI2 could protect the extracellular matrix of cancer cells from degradation and tumor invasion [14]. The loss of TFPI2 function might enhance the invasive potential of neoplastic cells in several cancers [15]. TFPI2 hypermethylation has been observed in CRC [16-18], GC [19-21], hepatocellular carcinoma [22], pancreatic cancer [23], and cervical cancer [15]. Interestingly, TFPI2 methylation disappeared in the serum of CRC patients after curative surgery [24], indicating a close correlation between TFPI2 methylation and CRC occurrence.

In this study, we used a quantitative methylation specific PCR (qMSP) method to measure TFPI2 methylation in GC and CRC tissues of Chinese Han patients, since the low sensitivity and specificity in the MSP method was still the main obstacle to the clinical application of TFPI2 methylation [16, 25]. The aim of this study was to evaluate whether cytosine-phosphateguanine $(\mathrm{CpG})$ island methylation of TFPI2 could serve as a valuable biomarker in $\mathrm{GC}$ and $\mathrm{CRC}$.

\section{RESULTS}

In the current study, we recruited 114 GC patients, 80 CRC patients and 22 non-tumor individuals to investigate the role of TFPI2 methylation on the detection of GC and CRC. There were two Methyl450 CpG sites (cg12973591 and cg22799321) located in the tested fragment (88 bp, hg38, chr7:93890180-93890267, Figure 1A). Meanwhile, Sanger sequencing showed that the amplified fragment matched the target sequence (Figure 1B).

Our data showed that TFPI2 methylation in tumor tissues was significantly higher than that in paired adjacent tissues [GC: $29.940 \%(15.472 \%, 47.295 \%)$ versus $12.785 \%$ (9.678\%, 16.575\%), $P<0.001$; CRC: $26.930 \%$ (8.478\%, $63.145 \%)$ versus $5.420 \%(1.345 \%, 16.638 \%), P<0.00001$; Figure 2A]. The methylation levels of TFPI2 in colorectal tumor tissues were significantly higher than those in colorectal normal tissues [26.930\% $(8.478 \%, 63.145 \%)$ versus $0.002 \%(0.001 \%, 0.054 \%), P<0.00001$, Figure $2 \mathrm{~A}]$. These data supported the previous findings that TFPI2 hypermethylation could be a potential novel biomarker for GC and CRC [18]. Then,TFPI2 hypermethylation was found in 85 out of $114 \mathrm{GC}$ tissues and 61 out of 80
CRC tissues. TFPI2 hypermethylation was a risk factor for $\mathrm{GC}$ and $\mathrm{CRC}$ [GC: $\mathrm{OR}=8.591$ (4.733-15.593); CRC: $\mathrm{OR}=10.307$ (4.976-21.351)]. Subsequently, we examined the correlation between TFPI2 methylation and the clinicopathological features of GC and CRC patients. However, no statistically significant correlation was found with age, gender, differentiation, lymph node metastasis, stage and tumor size (Table 1). We also compared the TFPI2 methylation levels between GC and CRC tumor samples, and no significant difference was found $(P=0.569)$. Our results didn't support TFPI2 hypermethylation as a differential biomarker between GC and CRC.

Since TFPI2 methylation was different in GC and CRC patients (85/114 in GC and 61/80 in CRC), we separately estimated the diagnostic value of TFPI2 methylation in GC and CRC. In GC, TFPI2 hypermethylation yielded an area under the curve (AUC) of 0.762 (95\% CI: $0.696-0.828)$ with a sensitivity of $68 \%$, a specificity of $83 \%$, a positive predictive value (PPV) of $74 \%$, and a negative predictive value (NPV) of $74 \%$ (Figure 3A). In CRC, TFPI2 hypermethylation yielded an AUC of 0.759 (95\% CI: $0.685-0.834)$ with a sensitivity of $61 \%$, a specificity of $84 \%$, a PPV of $76 \%$, and a NPV of 76\% (Figure 3B). Notably, TFPI2 hypermethylation yielded a high AUC of 1.00 (sensitivity: 100\%; specificity: $100 \%$ ) in CRC by comparing with the methylation level of TFPI2 between CRC and colorectal normal tissues (Figure 3C). Moreover, TFPI2 hypermethylation yielded a combined AUC of 0.753 (95\% CI: 0.703-0.802) with a sensitivity of $66 \%$ and a specificity of $82 \%$ (Figure 3D).

Similarly, TCGA data also supported TFPI2 hypermethylation as a promising diagnostic marker in GC and CRC. In GC, TFPI2 hypermethylation yielded an AUC of 0.883 (95\% CI: $0.809-0.957)$ with a sensitivity of $86 \%$ and a specificity of $89 \%$ (Figure 3E). In CRC, TFPI2 hypermethylation yielded an AUC of 0.967 (95\% CI: $0.929-1.000)$ with a sensitivity of $91 \%$ and a specificity of 100\% (Figure 3F). Moreover, TFPI2 hypermethylation yielded a combined AUC of 0.921 (95\% CI: 0.876-0.966) with a sensitivity of $79 \%$ and a specificity of $100 \%$ (Figure 3G).

In order to check whether TFPI2 $\mathrm{CpG}$ island region $(+538$ bp to $+938 \mathrm{bp})$ was able to regulate gene expression, we performed a dual-luciferase reporter assay. The results of luciferase detection after transfection showed that the transcriptional activity of recombinant pGL3TFPI2 plasmid was significantly higher compared to the pGL3-Basic control vector (fold change $=5, P=0.005$, Figure 2B).

The Cancer Genome Atlas (TCGA) database also validated the percentage of methylated reference (PMR) of TFPI2 gene in tumor tissues was higher than that in non-tumor adjacent tissues. Our data mining study showed that the relative methylation level was significantly higher in GC and CRC tissues (Figure 4A and 4B). Moreover, TFPI2 methylation was inversely correlated with gene 
Table 1: Association of TFPI2 methylation with clinical characteristics in gastric cancer and colorectal cancer patients

\begin{tabular}{|c|c|c|c|c|c|}
\hline \multirow{2}{*}{$\begin{array}{c}\text { Clinical } \\
\text { characteristics }\end{array}$} & \multirow{2}{*}{ Variable } & \multicolumn{2}{|c|}{ Gastric cancer patients } & \multicolumn{2}{|c|}{ Colorectal cancer patients } \\
\hline & & Numbers, PMR & $P$ Value & Numbers, PMR & $P$ Value \\
\hline \multirow{2}{*}{ Age } & $\leq 60$ & $66,29.485(16.668,47.478)$ & \multirow{2}{*}{0.754} & $42,29.845(8.565,49.010)$ & \multirow{2}{*}{0.343} \\
\hline & $>60$ & $48,31.685(14.290,48.015)$ & & $38,23.720(8.300,94.095)$ & \\
\hline \multirow[b]{2}{*}{ Gender } & Male & $78,28.260(15.125,48.570)$ & \multirow[b]{2}{*}{0.804} & $51,23.260(6.990,52.010)$ & \multirow[b]{2}{*}{0.18} \\
\hline & Female & $36,32.725(16.538,46.143)$ & & $29,34.390(12.680,65.935)$ & \\
\hline \multirow{2}{*}{ Differentiation } & Poorly differentiated & $67,31.020(16.270,45.770)$ & \multirow{2}{*}{0.653} & $15,11.460(4.130,48.770)$ & \multirow{2}{*}{$0.209^{\mathrm{a}}$} \\
\hline & Moderately + Well differentiated & $47,29.700(14.600,53.890)$ & & $48,32.160(11.280,65.990)$ & \\
\hline \multirow{2}{*}{ Disease stage } & $\mathrm{I}+\mathrm{II}$ & $18,20.935(9.700,38.138)$ & \multirow{2}{*}{0.126} & $41,24.460(9.945,53.230)$ & \multirow{2}{*}{0.867} \\
\hline & $\mathrm{III}+\mathrm{IV}$ & $96,32.050(16.913,48.495)$ & & $39,27.530(6.990,67.350)$ & \\
\hline \multirow{2}{*}{ Tumor Size } & $\leq 6 \mathrm{~cm}$ & $69,29.860(15.415,52.230)$ & \multirow{2}{*}{0.754} & $58,23.120(6.753,53.160)$ & \multirow{2}{*}{0.202} \\
\hline & $>6 \mathrm{~cm}$ & $45,30.020(15.435,45.660)$ & & $22,34.930(13.298,68.030)$ & \\
\hline \multirow{2}{*}{$\begin{array}{l}\text { Lymph nodes } \\
\text { metastasis }\end{array}$} & Positive & $97,31.020(16.535,47.670)$ & \multirow{2}{*}{0.599} & $43,32.580(10.910,74.110)$ & \multirow{2}{*}{0.193} \\
\hline & Negative & $17,22.980(11.585,48.595)$ & & $37,22.980(6.300,48.650)$ & \\
\hline
\end{tabular}

PMR stands for the percentage of methylated reference, and data were presented as median (interquartile range). $P$ value is calculated by Spearman test. a: The information of two cases' differentiation was lost.

expression in both GC and CRC (cg12973591, GC: $\mathrm{r}=-0.447, \mathrm{CRC}: \mathrm{r}=-0.225 ; \operatorname{cg} 22799321, \mathrm{GC}:$ $\mathrm{r}=-0.426$, CRC: $\mathrm{r}=-0.190)$. Besides, using the data from TCGA, we calculated the correlation between the 10 CG sites in the CpG island (cg12973591, $\operatorname{cg} 22799321$, cg20230721, cg17338208, cg26739865, cg24531255, cg23686014, cg23141855, cg14377593, cg2241153) in $\mathrm{CRC}$ tissues and non-tumor tissues, respectively. High correlation was observed between methylation levels of these $10 \mathrm{CG}$ sites $(P<0.00001$, data not shown). We also calculated the correlation between the 4 CG sites in the CpG island (cg22799321, cg23686014, cg23141855, and $\operatorname{cg} 14377593)$ in GC tissues and non-tumor tissues, respectively. High correlation was also observed among the methylation levels of these 4 CG sites $(P<0.00001$, data not shown).

Using the data of Gene Expression Omnibus (GEO) database (GSE32275), we focused on 5 CG sites (cg22799321, cg23686014, cg23141855, cg14377593, and cg19784477) in the $\mathrm{CpG}$ island. Our results showed that DNA methylation level dropped after 5'-AZAdeoxycytidine treatment (Supplementary Figure 1). Using the data of GEO database (GSE32323), we found that TFPI2 expression in three CRC cell lines (HCT116, RKO and SW480) was increased after 5'-AZA-deoxycytidine treatment (fold change $>1.37$, Figure $4 \mathrm{C}$ ). Therefore, TFPI2 was likely to be hypermethylated in the cell lines, which exerted a potential suppressor on gene expression.

\section{DISCUSSION}

In the current study, we compared the methylation level of TFPI2 among the GC and CRC and the adjacent non-tumor tissues, and colorectal normal tissues. TFPI2 hypermethylation was found in 85 out of $114(75 \%)$ GC tissues and 61 out of 80 (76\%) CRC tissues. Similarly, 52 out of $59(88 \%)$ GC tissues and 45 out of $45(100 \%)$ CRC tissues existed TFPI2 hypermethylation in TCGA database. TFPI2 methylation levels in CRC tissues were also significantly higher than those in colorectal normal tissues. Further data mining study of GEO data indicated that TFPI2 was likely to be hypermethylated in the CRC cell lines. The above findings suggested an important role of TFPI2 hypermethylation in the detection of GC and CRC.

In addition, although TFPI2 hypermethylation has been reported in many human cancers, there existed some highlights in our study. Firstly, most of studies applied MSP method, which is a qualitative approach with a low sensitivity in methylation detection [26]. We have chosen a quantitative method which has been considered as a more suitable application in molecular diagnosis $[16,27]$. Secondly, previous studies were involved with a relative fewer GC samples (ranging from 18 to 73 ) [19, 20]. In contrast, our GC sample size is 114. Thirdly, we further evaluated the regulation mechanism of TFPI2 methylation on gene expression. A dual-luciferase reporter gene assay showed an enhanced transcriptional activity of TFPI2 cloned fragment. Moreover, GEO data indicated that TFPI2 expression was significantly increased in three CRC cell lines (HCT116, PKO and SW480) after 5'-AZAdeoxycytidine treatment. Our results helped us gain an insight into the role of TFPI2 methylation in GC and CRC diagnosis.

Gastrointestinal malignant cancer was a genetically and phenotypically heterogeneous disease. Several distinct 
types of biomarkers have been studied in the diagnosis of cancer. Unfortunately, early detection of gastrointestinal malignant cancer has still been hindered by the absence of effective biomarkers. For example, carcinoembryonic antigen (CEA), as one of the most widely used tumor markers worldwide, played a role in cancer invasion and metastasis [28]. Fletcher has pointed out that CEA sensitivity and specificity were not high, particularly for early stages of disease, such as a sensitivity of $36 \%$ and a specificity of $87 \%$ in screening for colorectal cancer [29]. And Hibi et al. showed that the detection rate of colorectal cancer by using TFPI2 methylation, CEA and CA19-9 was $18 \%, 33 \%$ and $17 \%$, respectively [30]. Combined use of AFP, CEA, CA125 and CA19-9 for the diagnosis of GC had a relatively low AUC of 0.667 (sensitivity: 0.403; specificity: 0.932) [31]. Thus, conventional plasma proteins may lack enough sensitivity in clinical detection of GC and CRC. Epigenetic biomarkers may serve as a tool to diagnose cancer and predict therapeutic effect and prognosis [32]. The methylation status of a primary tumor could be discovered by qMSP in advance, which could be used as a cancer screening method in principle [16].

A

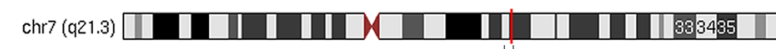
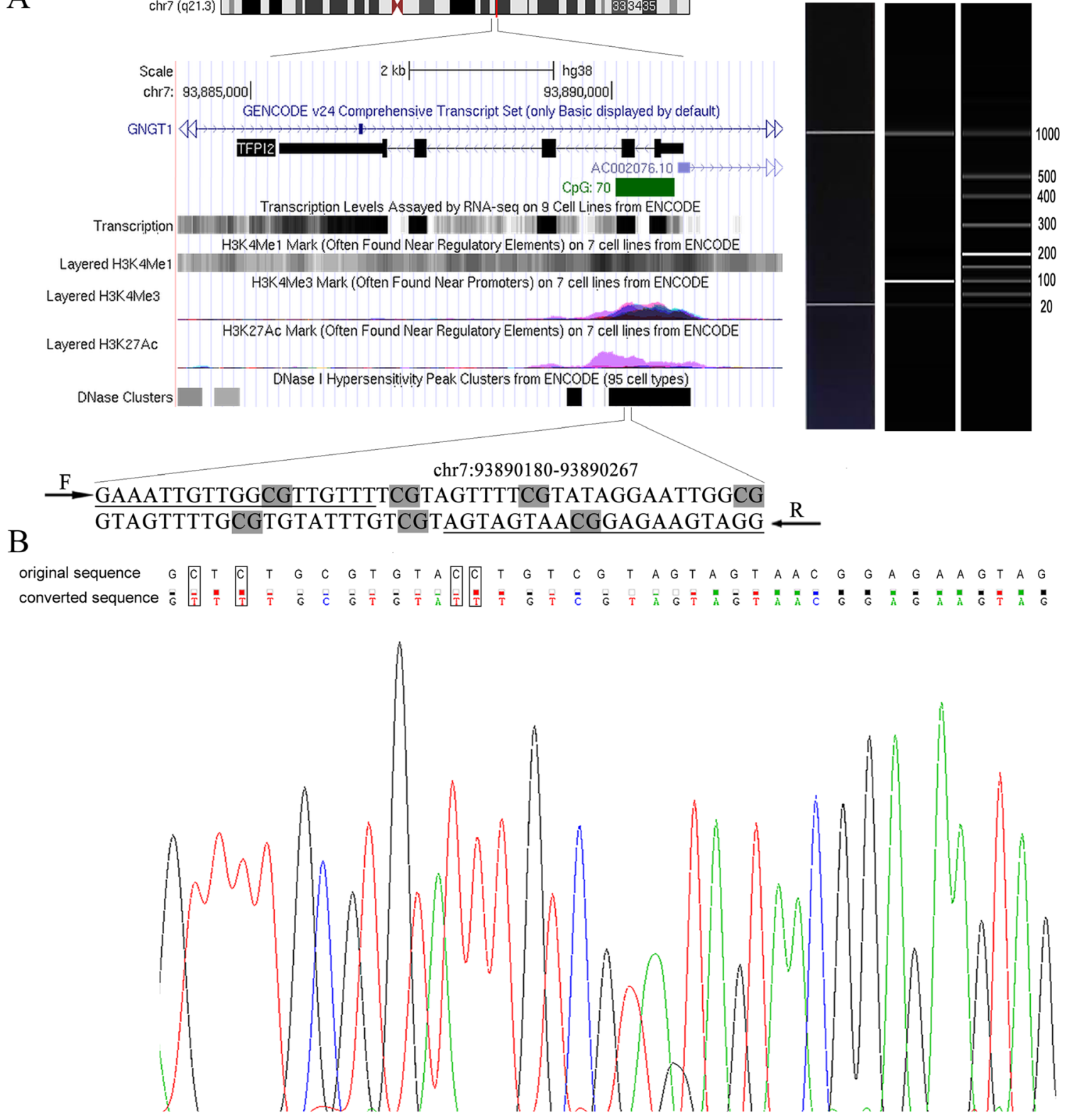

Figure 1: Target sequences on TFPI2 CpG island (CGI) region. (A) The genomic position and functional annotation of amplified fragment from UCSC genome browser according to human 2013 (GRCh38/hg38) assembly. The qMSP primers were underlined and seven CpG sites were in grey. F: forward primer; R: reverse primer. The picture on the right was the electrophoresis result of a representative qMSP product. (B) The top row of the sequence represented the original sequence, and the second row showed the converted sequence. And the framed base indicated that the cytosines were replaced by thymines ( $\mathrm{C}$ to T conversion) in bisulfite-treated DNA. 
Recently, many epigenetic biomarkers have been studied in cancer diagnosis, including $h M L H 1$, E-cadherin, $C D K N 2 A, C D K N 2 B$ and $A P C$ [33] in GC and SEPT9, SFRP2, THBD, SDC2, VIM and FBN1 in CRC [34]. Nevertheless, few biomarkers were actually used as auxiliary diagnosis of tumor in the clinic due to its low sensitivity. For example, Hibi et al. found only $10 \%$ GC samples and 18\% CRC samples exhibited TFPI2 methylation $[16,27]$. Our qMSP-based study showed that TFPI2 hypermethylation yielded a high AUC of 0.762 (sensitivity: 68\%; specificity: $83 \%$ ) in GC and 0.759 (sensitivity: 61\%; specificity: $84 \%$ ) in CRC. In addition, the biomarker should be required to distinguish the early/ late stages to accurately detect GC and CRC. According to our results, no statistically significant correlation was found between TFPI2 methylation and the stages of $\mathrm{GC}$ and $\mathrm{CRC}$ patients. Hence, combining methylation biomarkers with other biomarkers might further improve the diagnostic accuracy [14].

Studies have reported that GC and CRC share some molecular characteristics including microsatellite instability, hypermethylation of tumor suppressor genes, and genetic mutations $[35,36]$. And in addition, GC has very similar histopathology to CRC [37]. In the current study, we compared the TFPI2 methylation levels between $\mathrm{GC}$ and $\mathrm{CRC}$ tumor samples, and no significant difference was found $(P=0.569)$. Our results showed that TFPI2 hypermethylation has a similar diagnostic value for $\mathrm{GC}$ and CRC.

In the previous study, TFPI2 methylation could reduce expression in canine Diffuse Large B-cell lymphomas [38] and esophageal squamous cell carcinoma [39]. TFPI2 transcription restored in GC cells after treatment with 5'-AZA-deoxycytidine [20]. Our data

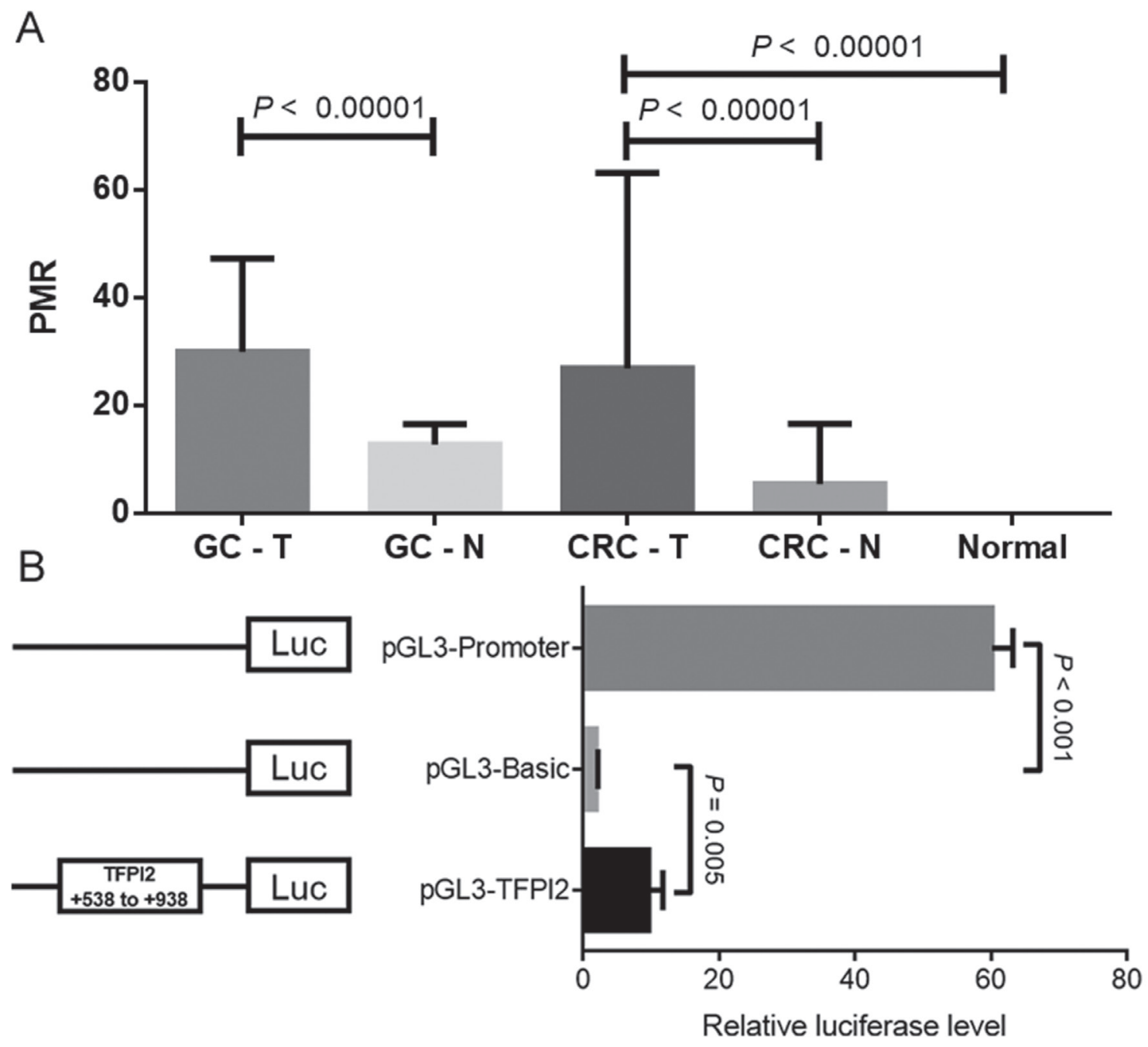

Figure 2: (A) Comparisons of TFPI2 methylation levels between tumor tissues and paired adjacent non-tumor tissues in GC patients and CRC patients. (B) Dual-luciferase reporter assay in HEK-293T cell line. The pGL3-Basic and pGL3-Promoter vectors were used as negative and positive controls, respectively. The pGL3-TFPI2 stood for the recombinant TFPI2 fragment ligated to pGL3-Basic vector. Relative luciferase activity was performed in quadruplicate. T stands for tumor tissues; $\mathrm{N}$ stands for adjacent non-tumor tissues. Normal stands for colorectal normal tissues from normal persons. Statistical values and the bar were presented as median with interquartile range. 
A

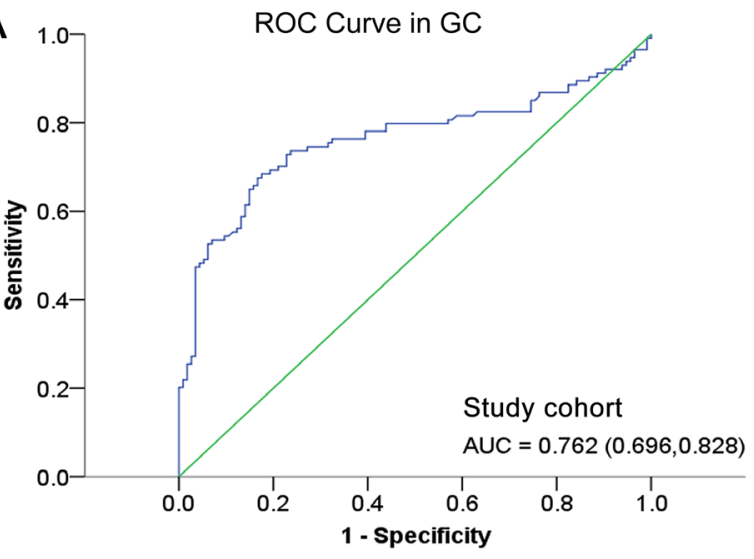

B

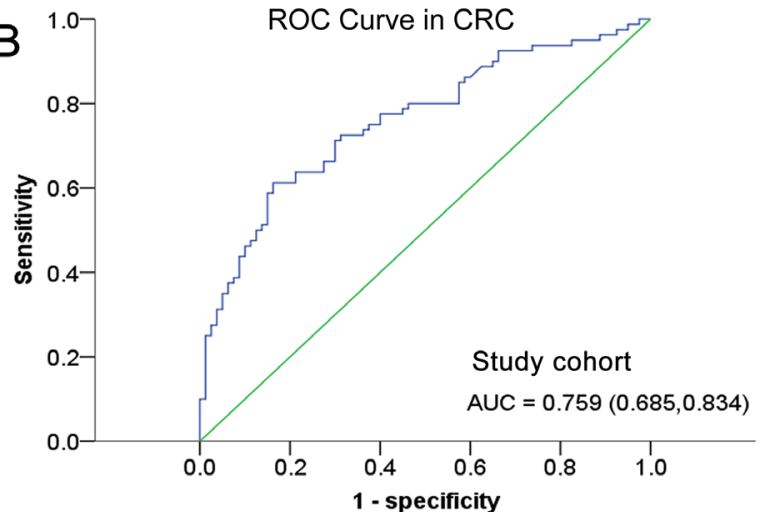

C

C ROC Curve in CRC and colorectal normal tissues
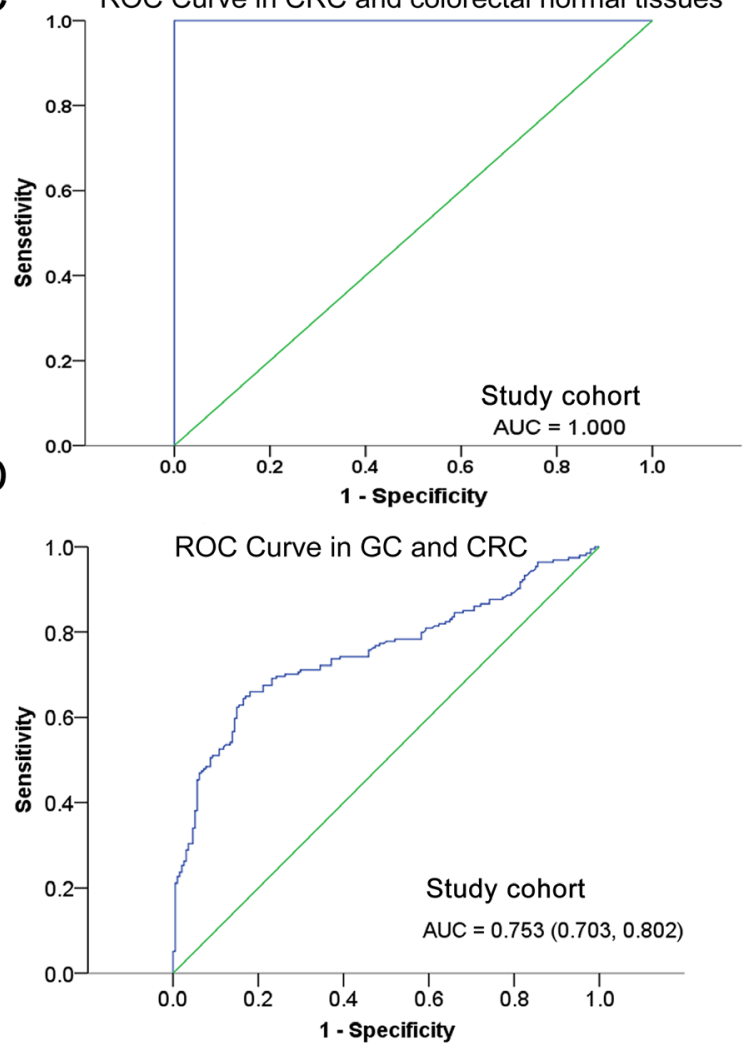
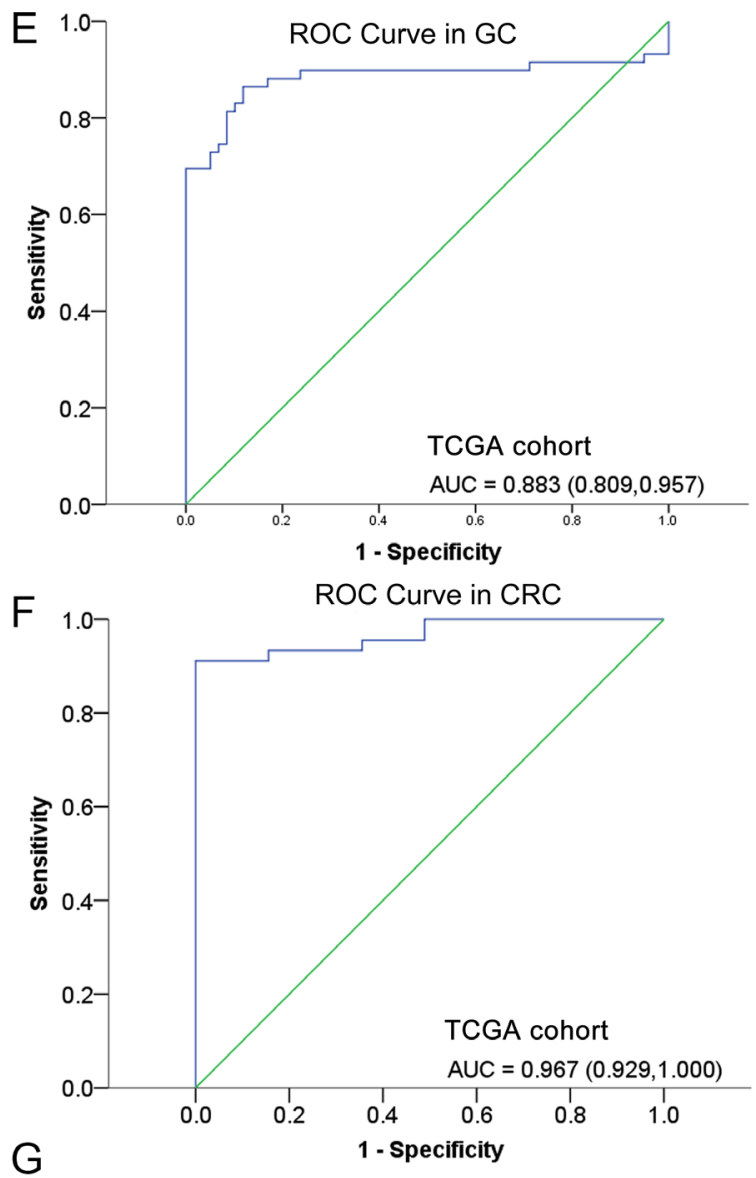

G

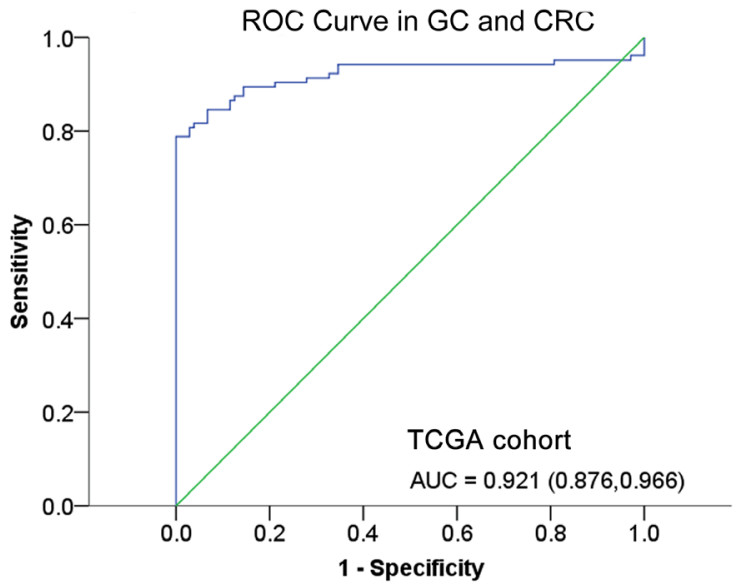

Figure 3: ROC curves for the diagnostic value of TFPI2 hypermethylation in (A) GC patients, (B) CRC patients, (C) CRC and colorectal normal tissues, and (D) combined individuals from study cohort, and (E) GC patients, (F) CRC patients, (G) combined individuals from TCGA cohort. ROC: receiver operating characteristic. AUC: area under the curve. 
mining of the public databases discovered there was an inverse correlation between TFPI2 methylation and gene expression in different cancers. Meanwhile, TFPI2 transcription in three CRC cell lines increased after 5'-AZA-deoxycytidine treatment in GEO database. In addition, our dual luciferase reporter assay showed a significantly higher activity in the recombinant strain with pGL3-TFPI2 plasmid, suggesting that the TFPI2 fragment was able to regulate gene expression.

Two familiar mechanisms of DNA methylation on transcriptional repression has been reported [40]. DNA methylation interfered with the combination of transcription factors and cis-element, which inhibited gene expression. In addition, methyl-CpG-binding protein altered the chromatin structure through recruiting the corepressor complex. According to UCSC Genome Browser, the selected fragment in the luciferase study overlapped with multiple transcription factor binding sites, including CCCTC binding factor (CTCF) which is known to play an unusual role in different aspects of gene regulation including promoter activation and repression, hormoneresponsive gene silencing, methylation-dependent chromatin insulation and genomic imprinting [41]. We hypothesized that TFPI2 was likely to be hypermethylated in GC and CRC, which exerted a potential suppressor on gene expression through inhibiting transcription factor binding. However, the exact regulatory mechanism should be explored in the future.

TFPI2 promoter was reported to be activated by $P 14 A R F$ in a p53-independent manner that relied upon c-JUN, SP1, and JNK activity, which could reduce aggressive phenotypes associated with necrotic tumors [42]. And the expression of TFPI2 had a decreasing trend with tumor progression of cervical cancer through
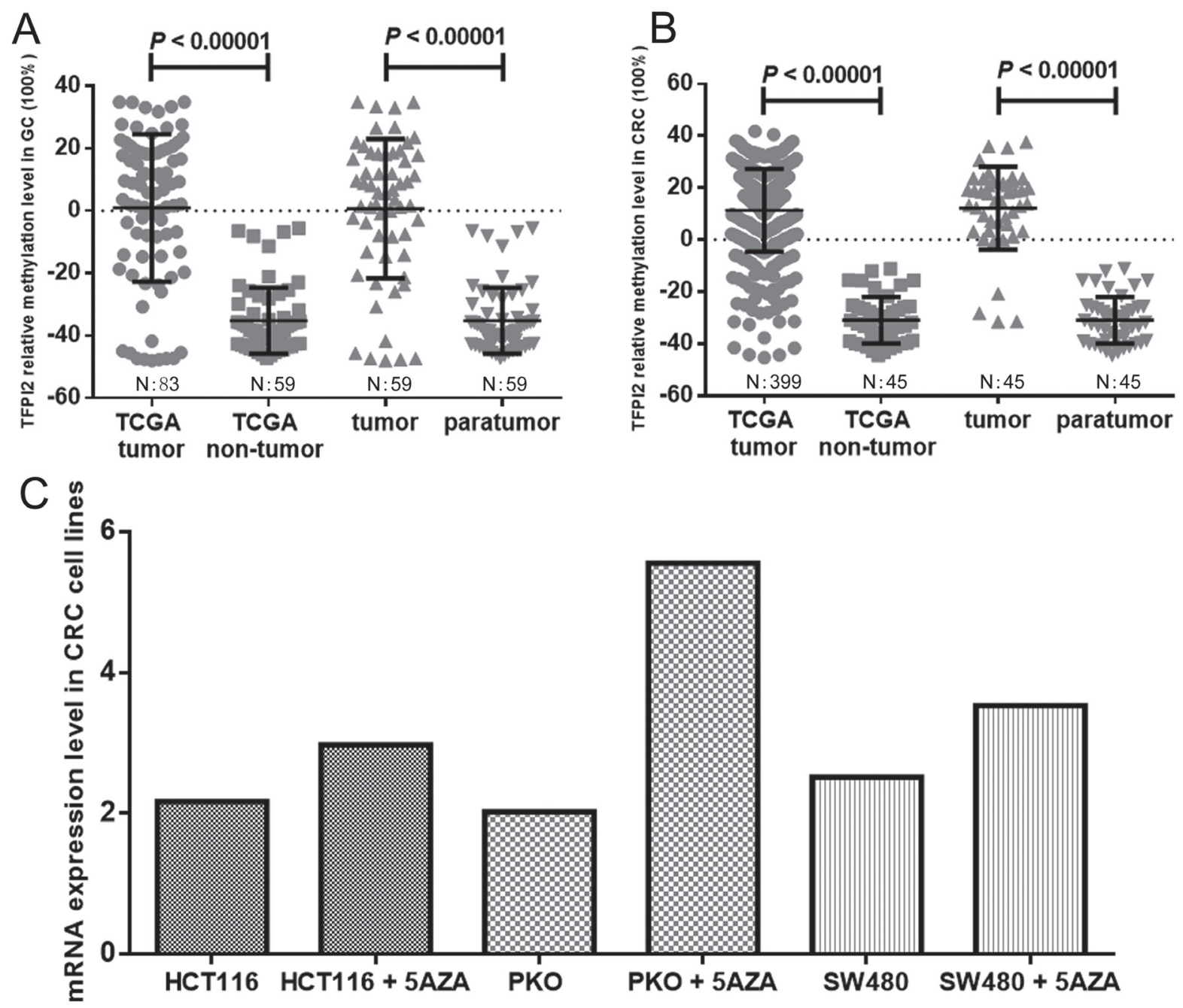

Figure 4: Analysis of TFPI2 gene methylation and mRNA expression in public databases. Comparisons of TFPI2 methylation levels between tumor tissues and adjacent non-tumor tissues in (A) GC patients and (B) CRC patients from TCGA database. The data of TCGA tumor and TCGA non-tumor were extracted from $83 \mathrm{GC}$ patients and 59 non-tumor patients in Figure 4A, 399 CRC patients and 45 non-tumor patients in Figure 4B, respectively. Tumor and paratumor were proceed from the same layer. N: number. Statistical values and the bars were presented as median with interquartile range. (C) The changes of mRNA expression levels in CRC cell lines (HCT116, PKO and SW480) with and without 5'-AZA-deoxycytidine treatment from GEO database (accession number GSE32323). 
inhibiting tumor cell apoptosis and angiogenesis [43]. Therefore, vascular thrombosis, hypoxia and necrosis induced by TFPI2 low-expression could be the main reasons for aggressive phenotypes in gastric and colorectal cancer pathways. Further study should be explored in gastric and colorectal cancer pathways.

What's more, TFPI2 lower-expression markedly inhibited its growth and metastasis in vivo by regulating pericellular extracellular matrix remodeling and angiogenesis [44]. Although TFPI2 was shown to reduce the cell proliferation rate in various human tumors, Lai et al. found that TFPI2 expression restoration after 5'-AZA-deoxycytidine treatment almost had no effect on suppressed cell proliferation and promoted cell apoptosis in vitro [45]. Therefore, the role of TFPI2 in cell proliferation and apoptosis may be complicated. Functional studies in vitro and in nude mice model should be performed in the future.

There are the following aspects of main limitations in our study to be noted. We only obtained tumor tissues in the current study. Since DNA methylation as a biomarker was more effective in serum than that in tissues [46], the diagnostic value of TFPI2 hypermethylation in serum should be assessed in the future. Due to the limitation of CRC cell lines, we only performed the transfection of TFPI2 CpG island region pGL3-Promoter vectors in HEK293T cell line, and future study in CRC cell lines should be performed to better understand the activity of the chosen promoter region. Due to the limitation in primer design, only $2 \mathrm{CpG}$ sites were evaluated to represent TFPI2 methylation. However, a more comprehensive research covering more regions among larger number of samples should be carried out in the future. In addition, although we utilized GEO data to further explore the potential regulatory mechanism between DNA methylation and gene expression, more studies should be carried out.

In summary, our studies suggested that TFPI2 hypermethylation might be an useful diagnostic biomarker for GC and CRC. Further studies on the detailed mechanisms of TFPI2 hypermethylation in the carcinogenesis should be explored.

\section{MATERIALS AND METHODS}

\section{Tissue samples}

Frozen tumor tissues and their paired adjacent normal tissues ( $5 \mathrm{~cm}$ away from the tumor lesion) were obtained from $114 \mathrm{GC}$ patients and $80 \mathrm{CRC}$ patients during surgery. We also collected 22 colorectal normal tissues after a diagnostic endoscopy. Pathological examination was performed for the diagnosis of all the patients. Hematoxylin and eosin (H\&E) stained slides were used to determine representative areas of invasive tumor. There are $80 \%$ of cancer cells presenting in each sample, which were examined by microscope. No tumor cells exist in the adjacent sample, and adjacent tissue was chosen from the same block as the tumor [47]. Besides, tumor and non-tumor samples proceed from the same layer. All the clinical information (including age, gender, disease stage, tumor differentiation and metastasis) was extracted from the medical records. Permission for the study was given by Human Research Ethics Committee at Shaoxing People's Hospital and Zhejiang Cancer Hospital. Written informed consent was obtained from each participant.

\section{DNA extraction and bisulphite conversion}

Isolated DNA was extracted from frozen tissues by E.Z.N.A. ${ }^{\text {TM }}$ Tissue DNA Kit (Omega Bio-Tek, Norcross, GA). Nanodrop2000 spectrophotometer (Thermal Scientific Co. Ltd., Wilmington, USA) was operated to measure DNA concentrations. And 500 ng DNA was used for bisulphite conversion. EZ DNA Methylation-Gold Kit тм (Zymo Research, Orange, USA) was used to convert unmethylated cytosines into uracils correspondingly, while the methylated cytosines remained in the reaction.

\section{Quantitative methylation specific polymerase chain reaction (qMSP) method}

$A C T B$ was chosen as the internal reference to correct the differences in both quality and quantity between samples. Human sperm DNA was methylated as the positive control by excess SssI methyltransferase (Thermo Fisher Scientiific, Uppsala, Sweden). All the bisulphite-modified DNA was used as the template in qMSP assays, which were carried out in 384-well plates in the LightCycler 480 (Roche, Basel, Switzerland). The final reaction system contained $10 \mu \mathrm{L}$ SYBR Green I Master (Roche, Basel, Switzerland), $0.5 \mu \mathrm{L}$ forward primer (10 $\mu \mathrm{m}), 0.5 \mu \mathrm{L}$ reverse primer $(10 \mu \mathrm{m}), 1.0 \mu \mathrm{L}$ templates and $8 \mu \mathrm{L} \mathrm{ddH_{2 }} \mathrm{O}$. PCR was conducted under the following conditions: 1 cycle at $95^{\circ} \mathrm{C}$ for $10 \mathrm{~min}$ followed by 45 cycles at $95^{\circ} \mathrm{C}$ for $20 \mathrm{sec}, 58^{\circ} \mathrm{C}$ for $20 \mathrm{sec}, 72^{\circ} \mathrm{C}$ for $30 \mathrm{sec}$; 1 cycle for the melting curve analysis at $95^{\circ} \mathrm{C}$ of $15 \mathrm{sec}$, $60^{\circ} \mathrm{C}$ of $1 \mathrm{~min}$; a final cooling stage at $40^{\circ} \mathrm{C}$ for $10 \mathrm{~min}$. The qMSP primer sequences were as follows: TFPI2 (88 bp), 5'-GAAATTGTTGGCGTTGTTT-3' (forward) and 5'-CCTACTTCTCCGTTACTACT-3' (reverse); ACTB (133 bp), 5'-TGGTGATGGAGGAGGTTTAGTAAGT-3' (forward)and 5'-AACCAATAAAACCTACTCCTCCCTT AA-3' (reverse). The PMR value of TFPI2 was used to represent gene expression. And the PMR in each sample was calculated by $2^{-\Delta \Delta \mathrm{Ct}}$ quantification approach, in which $\Delta \Delta \mathrm{Ct}=$ sample DNA $\left(\mathrm{Ct}\right.$ targetgene $\left.-\mathrm{Ct}{ }_{\mathrm{ACTB} \text { control }}\right)-$ fully methylated DNA $\left(\mathrm{Ct}_{\text {target gene }}-\mathrm{Ct}_{\text {ACTB controo }}\right)$ [48]. qMSP products were subjected to the Qsep100 DNA fragment analyzer (Bioptic, Taiwan, China), and they could be visualized by multiple objective straps in Gel-view format as previously described [49]. 


\section{Dual-luciferase reporter assay}

Dual-luciferase reporter system is a standard method that utilized firefly and renilla luciferase to study the promoter activity of target fragment [50]. Firefly and renilla luciferases are two commonly used reporters and are introduced into cells by co-transfecting cells with a luciferase reporter construct and an internal control vector to quantifiable luminescence [51]. The purpose of the dual luciferase assay in the current study was to show that the chosen regions might be functional in the regulation of gene expression. HEK293T cell line has a high success rate of transfection, and it is commonly used for plasmid transfection [52]. Therefore, we used the HEK293T cell line instead of CRC cell lines to perform the dualluciferase reporter assay. Human embryonic kidney HEK293T cell line was cultured as described previously [53]. A fragment of TFPI2 CpG island ( +538 bp to +938 bp) was chemically synthesized and was digested with XhoI and KpnI (New England Biolabs, Ipswich, MA). The target DNA fragment, purified by Cycle Pure Kit (Omega, Norcross, GA, USA), was ligated to pGL3-Basic vector using DNA Ligation Kit (TaKaRa, Japan). The empty pGL3-Basic vector (Promega, Madison city, WI, USA) was used as the negative control, and the pGL3Basic vector containing an SV40 promoter upstream of the luciferase gene, was used as the positive control.

Human HEK293T cells in the exponential growth phase were rinsed twice with phosphate buffer saline (PBS), treated with trypsin, and supplemented with dulbecco's minimum essential medium (DMEM) with $10 \%$ fatal bovine serum (FBS). The cells were calculated on 24-well plates. After 12 hours, $0.5 \times 10^{5}$ cells per well were transfected with recombinant plasmid according to the manufacturer's protocol (TransLipid HL Transfection Reagent, TransGen Biotech, Beijing, China). After 4-8 hours, medium was changed by fresh DMEM with $10 \%$ FBS. After 36 hours of 293T cells transfection, renilla and firefly luciferase activity was measured by SpectraMax 190 (Molecular Devices, Sunnyvale, USA). Reporter gene activity was assessed according to the manufacturer's protocol (Dual-Luciferase ${ }^{\mathbb{R}}$ Reporter Assay Systems, Promega, Madison city, WI, USA).

\section{Data mining study}

We extracted the $27 \mathrm{~K}$ array data of $83 \mathrm{GC}$ patients and $450 \mathrm{~K}$ array data of $399 \mathrm{CRC}$ patients from the TCGA database. First, we compared the TFPI2 methylation levels between the $83 \mathrm{GC}$ tissues and 59 non-tumor tissues. And TFPI2 methylation levels also were compared between the $399 \mathrm{CRC}$ tissues and 45 non-tumor tissues. Next, 59 paired GC samples and 45 paired CRC samples were chosen to validate the results.

DNA methylation is a reversible biochemical process [54], and 5'-AZA-deoxycytidine could serve as an inhibitor of DNA methylation [55]. 5'-AZA-deoxycytidine treatment is often used to restore the expression levels of genes which were expressed in low levels due to their hypermethylated promoters [56]. We retrieved the expression data derived from the Gene Expression Omnibus (GEO) database (www.ncbi.nlm.nih.gov/geo, accession no. GSE32323). We compared on the expression changes of TFPI2 in three CRC cell lines (HCT116, RKO and SW480) with and without 5'-AZA-deoxycytidine treatment.

\section{Statistical analysis}

All the data were analyzed by SPSS 18.0 software (SPSS Inc, Chicago, IL, USA) and GraphPad Prism 5.0 Software (GraphPad Software, La Jolla, CA). Data were presented as median (interquartile range). A nonparametric Mann-Whitney $U$ test was used to assess the methylation differences between tumor tissues and normal tissues. Spearman's correlation was used to evaluate the correlation between TFPI2 methylation level and clinical characteristics. Receiver operating characteristic (ROC) analysis showed the diagnostic value of TFPI2 methylation. A two-side $P<0.05$ was defined to be statistically significant.

\section{Abbreviations}

TFPI2, tissue factor pathway inhibitor 2; GC, gastric cancer; CRC, colorectal cancer; qMSP, quantitative methylation-specific polymerase chain reaction; PMR, percent of methylated reference; $\mathrm{CpG}$, cytosine-phosphateguanine; PPV, positive predictive value; NPV, negative predictive value; GEO, Gene Expression Omnibus; PBS, phosphate buffer saline; DMEM, dulbecco's minimum essential medium; FBS, fatal bovine serum; HM27K, Illumina Human Methylation 27K; HM450K, Illumina Human Methylation 450K; TCGA, The Cancer Genome Atlas; RMA, robust multi-array average; ROC, Receiver operating characteristic.

\section{Author contributions}

Conceived and designed the experiments: HH, SD and JY. Performed the experiments: XY, YJ, YY and BL. Analyzed the data: XC, JL and CW. Contributed reagents/ materials/analysis tools: DW, JY, CZ, YY and JZ. Wrote the paper: $\mathrm{HH}, \mathrm{XC}$ and SD.

\section{ACKNOWLEDGMENTS}

The research is supported by grants from National Natural Science Foundation of China (81371469), Natural Science Foundation of Zhejiang Province (LY14H160008), Clinical Research Foundation of Zhejiang Province Medical Association (2015ZYC-A77), K. C. Wong Magna Fund in Ningbo University and 
Science and Technology Plan Project of Shaoxing (grant no. 2015B70069).

\section{CONFLICTS OF INTEREST}

The authors declare no conflicts of interest.

\section{REFERENCES}

1. Bishak YK, Payahoo L, Osatdrahimi A, Nourazarian A. Mechanisms of cadmium carcinogenicity in the gastrointestinal tract. Asian Pac J Cancer Prev. 2015; 16:9-21.

2. Chen W, Zheng R, Baade PD, Zhang S, Zeng H, Bray F, Jemal A, Yu XQ, He J. Cancer statistics in China, 2015. CA Cancer J Clin. 2016; 66:115-32. https://doi.org/10.3322/ caac. 21338 .

3. Ye L, Lin ST, Mi YS, Liu Y, Ma Y, Sun HM, Peng ZH, Fan JW. Overexpression of LARP1 predicts poor prognosis of colorectal cancer and is expected to be a potential therapeutic target. Tumour Biol. 2016; 37:14585-94. https:// doi.org/10.1007/s13277-016-5332-3.

4. Kanda M, Shimizu D, Fujii T, Sueoka S, Tanaka Y, Ezaka K, Takami H, Tanaka H, Hashimoto R, Iwata N, Kobayashi D, Tanaka C, Yamada S, et al. Function and diagnostic value of Anosmin-1 in gastric cancer progression. Int J Cancer. 2016; 138:721-30. https://doi.org/10.1002/ijc.29803.

5. Llorca-Cardeñosa MJ, Fleitas T, Ibarrola-Villava M, PeñaChilet M, Mongort C, Martinez-Ciarpaglini C, Navarro L, Gambardella V, Castillo J, Roselló S, Navarro S, Ribas G, Cervantes A. Epigenetic changes in localized gastric cancer: the role of RUNX3 in tumor progression and the immune microenvironment. Oncotarget. 2016; 7:63424-36. https:// doi.org/10.18632/oncotarget.11520.

6. Luo X, Huang R, Sun H, Liu Y, Bi H, Li J, Yu H, Sun J, Lin S, Cui B, Zhao Y. Methylation of a panel of genes in peripheral blood leukocytes is associated with colorectal cancer. Sci Rep. 2016; 6:29922. https://doi.org/10.1038/ srep29922.

7. Li M, Gao F, Xia Y, Tang Y, Zhao W, Jin C, Luo H, Wang J, Li Q, Wang Y. Filtrating colorectal cancer associated genes by integrated analyses of global DNA methylation and hydroxymethylation in cancer and normal tissue. Sci Rep. 2016; 6:31826. https://doi.org/10.1038/srep31826.

8. Li L, Li C, Mao H, Du Z, Chan WY, Murray P, Luo B, Chan AT, Mok TS, Chan FK, Ambinder RF, Tao Q. Epigenetic inactivation of the CpG demethylase TET1 as a DNA methylation feedback loop in human cancers. Sci Rep. 2016; 6:26591. https://doi.org/10.1038/srep26591.

9. Semaan A, van Ellen A, Meller S, Bergheim D, Branchi V, Lingohr P, Goltz D, Kalff JC, Kristiansen G, Matthaei H, Pantelis D, Dietrich D. SEPT9 and SHOX2 DNA methylation status and its utility in the diagnosis of colonic adenomas and colorectal adenocarcinomas. Clin Epigenetics. 2016; 8:100. https://doi.org/10.1186/s13148016-0267-5.
10. Bagci B, Sari M, Karadayi K, Turan M, Ozdemir O, Bagci G. KRAS, BRAF oncogene mutations and tissue specific promoter hypermethylation of tumor suppressor SFRP2, DAPK1, MGMT, HIC1 and p16 genes in colorectal cancer patients. Cancer Biomark. 2016; 17:133-43. https://doi. org/10.3233/CBM-160624.

11. Pimson C, Ekalaksananan T, Pientong C, Promthet S, Putthanachote N, Suwanrungruang K, Wiangnon S. Aberrant methylation of PCDH10 and RASSF1A genes in blood samples for non-invasive diagnosis and prognostic assessment of gastric cancer. PeerJ. 2016; 4:e2112. https:// doi.org/10.7717/peerj.2112.

12. Choi B, Han TS, Min J, Hur K, Lee SM, Lee HJ, Kim YJ, Yang HK. MAL and TMEM220 are novel DNA methylation markers in human gastric cancer. Biomarkers. 2017; 22:35-44. https://doi.org/10.1080/1354750X.2016.1201542.

13. Rollin J, Iochmann S, Blechet C, Hube F, Regina S, Guyetant S, Lemarie E, Reverdiau P, Gruel Y. Expression and methylation status of tissue factor pathway inhibitor-2 gene in non-small-cell lung cancer. Br J Cancer. 2005; 92:775-83. https://doi.org/10.1038/sj.bjc.6602298.

14. Gerecke C, Scholtka B, Lowenstein Y, Fait I, Gottschalk U, Rogoll D, Melcher R, Kleuser B. Hypermethylation of ITGA4, TFPI2 and VIMENTIN promoters is increased in inflamed colon tissue: putative risk markers for colitisassociated cancer. J Cancer Res Clin Oncol. 2015; 141:2097-107. https://doi.org/10.1007/s00432-015-1972-8.

15. Dong Y, Tan Q, Tao L, Pan X, Pang L, Liang W, Liu W, Zhang W, Li F, Jia W. Hypermethylation of TFPI2 correlates with cervical cancer incidence in the Uygur and Han populations of Xinjiang, China. Int J Clin Exp Pathol. 2015; 8:1844-54.

16. Hibi K, Goto T, Shirahata A, Saito M, Kigawa G, Nemoto $\mathrm{H}$, Sanada Y. Detection of TFPI2 methylation in the serum of gastric cancer patients. Anticancer Res. 2011; 31:3835-8.

17. Zhang J, Yang S, Xie Y, Chen X, Zhao Y, He D, Li J. Detection of methylated tissue factor pathway inhibitor 2 and human long DNA in fecal samples of patients with colorectal cancer in China. Cancer Epidemiol. 2012; 36:737. https://doi.org/10.1016/j.canep.2011.04.006.

18. Glockner SC, Dhir M, Yi JM, McGarvey KE, Van Neste L, Louwagie J, Chan TA, Kleeberger W, de Bruine AP, Smits KM, Khalid-de Bakker CA, Jonkers DM, Stockbrugger RW, et al. Methylation of TFPI2 in stool DNA: a potential novel biomarker for the detection of colorectal cancer. Cancer Res. 2009; 69:4691-9. https://doi.org/10.1158/0008-5472. CAN-08-0142.

19. Hibi K, Goto T, Kitamura YH, Sakuraba K, Shirahata A, Mizukami H, Saito M, Ishibashi K, Kigawa G, Nemoto $\mathrm{H}$, Sanada Y. Methylation of the TFPI2 gene is frequently detected in advanced gastric carcinoma. Anticancer Res. 2010; 30:4131-3.

20. Takada H, Wakabayashi N, Dohi O, Yasui K, Sakakura C, Mitsufuji S, Taniwaki M, Yoshikawa T. Tissue factor pathway inhibitor 2 (TFPI2) is frequently silenced by 
aberrant promoter hypermethylation in gastric cancer. Cancer Genet Cytogenet. 2010; 197:16-24. https://doi. org/10.1016/j.cancergencyto.2009.11.004.

21. Jee CD, Kim MA, Jung EJ, Kim J, Kim WH. Identification of genes epigenetically silenced by $\mathrm{CpG}$ methylation in human gastric carcinoma. Eur J Cancer. 2009; 45:1282-93. https://doi.org/10.1016/j.ejca.2008.12.027.

22. Sun FK, Fan YC, Zhao J, Zhang F, Gao S, Zhao ZH, Sun Q, Wang K. Detection of TFPI2 methylation in the serum of hepatocellular carcinoma patients. Dig Dis Sci. 2013; 58:1010-5. https://doi.org/10.1007/s10620-012-2462-3.

23. Kisiel JB, Yab TC, Taylor WR, Chari ST, Petersen GM, Mahoney DW, Ahlquist DA. Stool DNA testing for the detection of pancreatic cancer: assessment of methylation marker candidates. Cancer. 2012; 118:2623-31. https://doi. org/10.1002/cncr.26558.

24. Hibi K, Goto T, Shirahata A, Saito M, Kigawa G, Nemoto $\mathrm{H}$, Sanada Y. Methylation of TFPI2 no longer detected in the serum DNA of colorectal cancer patients after curative surgery. Anticancer Res. 2012; 32:787-90.

25. Ma J, Yang Q, Deng C, Jin H, Gong W, Wang S, Chen H, Fan Y. [Screening and diagnostic value of the molecular markers of DNA methylation in colorectal neoplasma]. [Article in Chinese]. Zhonghua Wei Chang Wai Ke Za Zhi. 2015; 18:1149-53.

26. Malpeli G, Amato E, Dandrea M, Fumagalli C, Debattisti V, Boninsegna L, Pelosi G, Falconi M, Scarpa A. Methylationassociated down-regulation of RASSF1A and up-regulation of RASSF1C in pancreatic endocrine tumors. BMC Cancer. 2011; 11:351. https://doi.org/10.1186/1471-2407-11-351.

27. Hibi K, Goto T, Kitamura YH, Yokomizo K, Sakuraba K, Shirahata A, Mizukami H, Saito M, Ishibashi K, Kigawa G, Nemoto H, Sanada Y. Methylation of TFPI2 gene is frequently detected in advanced well-differentiated colorectal cancer. Anticancer Res. 2010; 30:1205-7.

28. Jessup JM, Thomas P. Carcinoembryonic antigen: function in metastasis by human colorectal carcinoma. Cancer Metastasis Rev. 1989; 8:263-80.

29. Fletcher RH. Carcinoembryonic antigen. Ann Intern Med. 1986; 104:66-73.

30. Hibi K, Goto T, Shirahata A, Saito M, Kigawa G, Nemoto $\mathrm{H}$, Sanada Y. Detection of TFPI2 methylation in the serum of colorectal cancer patients. Cancer Lett. 2011; 311:96100. https://doi.org/10.1016/j.canlet.2011.07.006.

31. He CZ, Zhang KH, Li Q, Liu XH, Hong Y, Lv NH. Combined use of AFP, CEA, CA125 and CA19-9 improves the sensitivity for the diagnosis of gastric cancer. BMC Gastroenterol. 2013; 13:87. https://doi.org/10.1186/1471-230X-13-87.

32. Verma M. The Role of Epigenomics in the Study of Cancer Biomarkers and in the Development of Diagnostic Tools. Adv Exp Med Biol. 2015; 867:59-80. https://doi. org/10.1007/978-94-017-7215-0_5.

33. Liu L, Cao L, Gong B, Yu J. Novel biomarkers for the identification and targeted therapy of gastric cancer. Expert
Rev Gastroenterol Hepatol. 2015; 9:1217-26. https://doi.or g/10.1586/17474124.2015.1072466.

34. Coppede F, Lopomo A, Spisni R, Migliore L. Genetic and epigenetic biomarkers for diagnosis, prognosis and treatment of colorectal cancer. World J Gastroenterol. 2014; 20:943-56. https://doi.org/10.3748/wjg.v20.i4.943.

35. Wu M, Semba S, Oue N, Ikehara N, Yasui W, Yokozaki H. BRAF/K-ras mutation, microsatellite instability, and promoter hypermethylation of hMLH1/MGMT in human gastric carcinomas. Gastric Cancer. 2004; 7:246-53. https:// doi.org/10.1007/s10120-004-0300-9.

36. Lee HS, Choi SI, Lee HK, Kim HS, Yang HK, Kang GH, Kim YI, Lee BL, Kim WH. Distinct clinical features and outcomes of gastric cancers with microsatellite instability. Mod Pathol. 2002; 15:632-40. https://doi.org/10.1038/ modpathol.3880578.

37. Lee HS, Hwang SM, Kim TS, Kim DW, Park DJ, Kang SB, Kim HH, Park KU. Circulating methylated septin 9 nucleic Acid in the plasma of patients with gastrointestinal cancer in the stomach and colon. Transl Oncol. 2013; 6:290-6.

38. Ferraresso S, Bresolin S, Arico A, Comazzi S, Gelain ME, Riondato F, Bargelloni L, Marconato L, Kronnie G, Aresu L. Epigenetic silencing of TFPI-2 in canine diffuse large B-cell lymphoma. PLoS One. 2014; 9:e92707. https://doi. org/10.1371/journal.pone.0092707.

39. Tsunoda S, Smith E, De Young NJ, Wang X, Tian ZQ, Liu JF, Jamieson GG, Drew PA. Methylation of CLDN6, FBN2, RBP1, RBP4, TFPI2, and TMEFF2 in esophageal squamous cell carcinoma. Oncol Rep. 2009; 21:1067-73.

40. Xiaoying C, Huadan Y, Qingxiao H, Annan Z, Linlin T, Shiwei D. [The effects of DNA methylation on the homeostasis in vascular diseases]. [Article in Chinese]. Yi Chuan. 2015; 37:221-32. https://doi.org/10.16288/j. yczz.14-327.

41. Rakha EA, Pinder SE, Paish CE, Ellis IO. Expression of the transcription factor CTCF in invasive breast cancer: a candidate gene located at 16q22.1. Br J Cancer. 2004; 91:1591-6. https://doi.org/10.1038/sj.bjc.6602144.

42. Zerrouqi A, Pyrzynska B, Brat DJ, Van Meir EG. P14ARF suppresses tumor-induced thrombosis by regulating the tissue factor pathway. Cancer Res. 2014; 74:1371-8. https:// doi.org/10.1158/0008-5472.CAN-13-1951.

43. Zhang Q, Zhang Y, Wang SZ, Wang N, Jiang WG, Ji YH, Zhang SL. Reduced expression of tissue factor pathway inhibitor-2 contributes to apoptosis and angiogenesis in cervical cancer. J Exp Clin Cancer Res. 2012; 31:1. https:// doi.org/10.1186/1756-9966-31-1.

44. Chand HS, Du X, Ma D, Inzunza HD, Kamei S, Foster D, Brodie S, Kisiel W. The effect of human tissue factor pathway inhibitor-2 on the growth and metastasis of fibrosarcoma tumors in athymic mice. Blood. 2004; 103:1069-77. https://doi.org/10.1182/blood-2003-06-1930.

45. Lai YH, He RY, Chou JL, Chan MW, Li YF, Tai CK. Promoter hypermethylation and silencing of tissue factor 
pathway inhibitor-2 in oral squamous cell carcinoma. J Transl Med. 2014; 12:237. https://doi.org/10.1186/s12967014-0237-7.

46. Ling ZQ, Lv P, Lu XX, Yu JL, Han J, Ying LS, Zhu X, Zhu WY, Fang XH, Wang S, Wu YC. Circulating Methylated XAF1 DNA Indicates Poor Prognosis for Gastric Cancer. PLoS One. 2013; 8:e67195. https://doi.org/10.1371/journal. pone.0067195.

47. Rauscher GH, Kresovich JK, Poulin M, Yan L, Macias V, Mahmoud AM, Al-Alem U, Kajdacsy-Balla A, Wiley EL, Tonetti D, Ehrlich M. Exploring DNA methylation changes in promoter, intragenic, and intergenic regions as early and late events in breast cancer formation. BMC Cancer. 2015; 15:816. https://doi.org/10.1186/s12885-015-1777-9.

48. Kristensen LS, Mikeska T, Krypuy M, Dobrovic A. Sensitive Melting Analysis after Real Time- Methylation Specific PCR (SMART-MSP): high-throughput and probefree quantitative DNA methylation detection. Nucleic Acids Res. 2008; 36:e42. https://doi.org/10.1093/nar/gkn113.

49. Chen X, Hu H, Liu J, Yang Y, Liu G, Ying X, Chen Y, Li B, Ye $\mathrm{C}, \mathrm{Wu} \mathrm{D}$, Duan S. FOXF2 promoter methylation is associated with prognosis in esophageal squamous cell carcinoma. Tumour Biol. 2017; 39. https://doi.org/10.1177/1010428317692230.

50. Xu YZ, Kanagaratham C, Jancik S, Radzioch D. Promoter deletion analysis using a dual-luciferase reporter system. Methods Mol Biol. 2013; 977:79-93. https://doi. org/10.1007/978-1-62703-284-1_7.
51. Wu GQ, Wang X, Zhou HY, Chai KQ, Xue Q, Zheng AH, Zhu XM, Xiao JY, Ying XH, Wang FW, Rui T, Xu LY, Zhang YK, et al. Evidence for transcriptional interference in a dual-luciferase reporter system. Sci Rep. 2015; 5:17675. https://doi.org/10.1038/srep17675.

52. Xue JH, Xu GF, Gu TP, Chen GD, Han BB, Xu ZM, Bjoras M, Krokan HE, Xu GL, Du YR. Uracil-DNA Glycosylase UNG Promotes Tet-mediated DNA Demethylation. J Biol Chem. 2016; 291:731-8. https://doi.org/10.1074/jbc. M115.693861.

53. Shen Z, Chen X, Li Q, Zhou C, Xu Y, Yu R, Ye H, Li J, Duan S. Elevated methylation of CMTM3 promoter in the male laryngeal squamous cell carcinoma patients. Clin Biochem. 2016; 49:1278-82. https://doi.org/10.1016/j. clinbiochem.2016.08.002.

54. Ramchandani S, Bhattacharya SK, Cervoni N, Szyf M. DNA methylation is a reversible biological signal. Proc Natl Acad Sci USA. 1999; 96:6107-12.

55. Farinha NJ, Shaker S, Lemaire M, Momparler L, Bernstein M, Momparler RL. Activation of expression of p15, p73 and E-cadherin in leukemic cells by different concentrations of 5-aza-2'-deoxycytidine (Decitabine). Anticancer Res. 2004; $24: 75-8$.

56. Momparler RL. Cancer epigenetics. Oncogene. 2003; 22:6479-83. https://doi.org/10.1038/sj.onc.1206774. 